and were similar in the 2 groups at the beginning of the study. Visual fields compared at the end of the third, sixth, and twelfth month of the trial were significantly improved in both groups. Relief of headache occurred after a mean of 3.75 and 3.3 months treatment. Papilledema began to regress after the second month. Side effects of topiramate included distal paresthesias and concentration difficulties; acetazolamide caused fatigue and distal paresthesias. A prominent weight loss (mean $9.75 \mathrm{~kg}$ ) in the topiramate group was significantly greater than in acetazolamide treated patients $(p<0.001)$. Both drugs were equally effective in treatment of $\mathrm{IIH}$. A placebo-controlled, double-blind trial of topiramate in IIH is recommended. (Celebisoy N, Gokcay F, Sirin H, Akyurekli O. Treatment of idiopathic intracranial hypertension: topiramate vs acetazolamide, an open-label study. Acta Neurol Scand November 2007;1 16:322-327). (Respond: Dr Nese Celebisoy, Department of Neurology, Ege University Medical School, Bornova, Izmir, 35100, Turkey. E-mail: nese.celebisoy(ajege.edu.tr).

COMMENT. Acetazolamide is the first-line drug in treatment of IIH. It acts by inhibiting carbonic anhydrase and reducing CSF production. Topiramate also inhibits carbonic anhydrase, and the mechanism of action in IIH may be similar to that of acetazolamide. The above patient cohort included some adolescents, but the majority were adults. Children with IIH differ from adults in a slight preponderance of male vs female and a much smaller incidence of obesity. Of 10 prepubertal pediatric patients studied at Children's Hospital Los Angeles, only one was obese, and the most common presenting signs were stiff neck, diplopia and strabismus. Papilledema and sixth nerve palsy resolved rapidly with acetazolamide. (Cinciripini GS et al. Am J Ophthalmol 1999;127:178-182). A 14-year-old male adolescent with IIH and almost daily headache responded within 2 to 4 weeks following topiramate therapy. (Palacio E et al. Headache 2004;44:436-437). The results of the Turkish study are of interest, and a trial of topiramate in children with IIH is warranted.

\title{
FAMILIAL BRAIN ARTERIOVENOUS MALFORMATIONS
}

Researchers at University Medical Centre Utrecht, the Netherlands, reviewed the literature on patients with familial brain arteriovenous malformations (BAVMs), and their age, sex, and clinical presentation were compared with those in population-based patients with sporadic BAVMs. A total of 53 patients were identified in 25 families, including 3 families at the Utrecht center. Mean age at diagnosis of familial cases of BAVM was 27 years (range 9 months to 58 years), and 8 years younger than sporadic cases. Sex incidence and presenting symptoms were similar in familial and sporadic cases. Male: female ratio was $1: 1$, and presenting symptoms were intracranial hemorrhage in $47 \%$, epilepsy $26 \%$, headache $9 \%$, and focal neurologic deficit in $2 \%$. Clinical anticipation was likely in families with BAVMs in successive generations; children born to a parent with AVM presented at a mean age 22 years younger (17 vs 39 years). (van Beijnum J, van der Worp HB, Schippers HM et al. Familial occurrence of brain arteriovenous malformations: a systematic review. J Neurol Neurosurg Psychiatry November 2007;78:1213-1217). (Respond: Dr Janneke van Beijnum, Department of Neurology and Neurosurgery, University Medical Centre Utrecht, GO3. 124, PO Box 85500, 3508 GA Utrecht, the Netherlands. E-mail: J.vanBeijnum(a) umcutrecht.nl). 
COMMENT. Familal BAVM is rare with only 25 families reported. Familial BAVMs present at a younger age than sporadic cases, and clinical anticipation of the disorder may occur in children with BAVM born to affected parents.

Presenting symptoms, surgical management and outcome of 39 pediatric cases of AVM are reviewed by Klimo P Jr et al. Child's Nerv Syst 2007;23:31-37; Ped Neur Briefs Jan 2007;21:1-2.

\section{SEIZURE DISORDERS}

\section{LONG-TERM OUTCOME OF NEONATAL SEIZURES}

Neonates with seizures prospectively diagnosed in a population-based setting in Newfoundland, Canada, between 1990 and 1995, were followed to a median age of 10 years. The incidence of epilepsy, and physical and cognitive impairments were analyzed for 62 term and 26 premature infants. Outcome was normal in 31 children $(35 \%) ; 36(41 \%)$ had neurodevelopmental impairments: and $21(24 \%)$ had died. The median age at death was 13 months; range 2 days to 14 years. Gestational age was a better predictor of outcome than birth weight. Normal outcome occurred more often in term than preterm infants $(\mathrm{p}=0.003)$. Postneonatal epilepsy developed in 27 (34\%), including $29 \%$ of term and $48 \%$ preterm infants. Median age at onset of epilepsy was 9 months in term and 4 months in preterm infants. Epilepsy complicating mental retardation (MR) and cerebral palsy (CP) was correlated with early death. CP occurred in $35 \%$ of those surviving infancy, MR in $32 \%$, and learning disorders in $23 \%$. Neonatal encephalopathy occurred in $42 \%$, usually following hypoxic-ischemic events. Infants with Sarnat stage III encephalopathy either died or had MR, $\mathrm{CP}$, and symptomatic epilepsy. Infections occurred in $19 \%$; outcome was normal in term infants with infection, but poor in premature infants or with CMV infection.

Abnormal neonatal EEGs, usually an abnormal background activity, correlated closely with poor outcome $(\mathrm{p}<0.0001)$. Other variables associated with a poor prognosis included cerebral dysgenesis, intraventricular hemorrhage, and need for multiple AEDs. Purely clonic seizures without facial involvement in term infants had a favorable prognosis, whereas generalized myoclonic seizures in preterm infants were associated with high mortality. Subtle seizures, generalized tonic seizures, and number of seizures were not predictive of outcome. The severity and timing of the brain insult are the major determinants of outcome. (Ronen GM, Buckley D, Penney S, Streiner DL. Long-term prognosis in children with neonatal seizures. Neurology Nov 6, 2007;69:1816-1822). (Reprints: Dr Gabriel M Ronen, HSC 3N11, 1200 Main Street West, Hamilton, Ontario, Canada L8N3Z5).

COMMENT. In an editorial, Dlugos D and Sirven JI (Prognosis of neonatal seizures. "It's the etiology, Stupid" - or is it? Neurology 2007;69:1812-1813) refer to the study by Mizrahi E and Kellaway P. (Neurology 1987;37:1837-1844) who found, using video-EEG, that focal clonic seizures and focal tonic seizures were consistently associated with ictal activity on EEG. In contrast, generalized myoclonic events showed ictal EEG correlation in $60 \%$ of cases. Motor automatisms (subtle seizures), generalized tonic events, and focal myoclonic jerks were not associated with EEG ictal activity, suggesting that these clinical events may not be epileptic. A distinction was made between clinical events that were epileptic and those associated with an encephalopathy but were not neonatal seizures. The 